\title{
Central vestibular syndrome in a red fox (Vulpes vulpes) with presumptive right caudal cerebral artery ischemic infarct and prevalent midbrain involvement
}

\author{
${\text { Mario Ricciardi }{ }^{1, *} \text {, Floriana Gernone }}^{1}$, Antonio De Simone ${ }^{2}$ and Pasquale Giannuzzi ${ }^{1}$ \\ 1 "Pingry" Veterinary Hospital, via Medaglie d'Oro 5, 70126 Bari, Italy \\ 2 "Chisimaio" Veterinary Clinic, via Chisimaio 32, 00199 Roma, Italy
}

\begin{abstract}
A wild young male red fox (Vulpes vulpes) was found in the mountainous hinterland of Rome (Italy) with a heavily depressed mental status and unresponsive to the surrounding environment. Neurological examination revealed depression, left circling, right head tilt, ventromedial positional strabismus and decreased postural reactions on the left side. Neurological abnormalities were suggestive of central vestibular syndrome. Two consecutive MRIs performed with 30 days interval were compatible with lacunar ischemic infarct in the territory of right caudal cerebral artery and its collateral branches. The lesion epicentre was in the right periaqueductal portion of the rostral mesencephalic tegmentum. Neuroanatomical and neurophysiological correlation between lesion localization and clinical presentation are discussed.
\end{abstract}

Keywords: Mesencephalon, Midbrain, MRI, Stroke, Vulpes vulpes.

\section{Introduction}

Cerebral ischemic stroke is a sudden interruption of arterial blood flow in a limited area of the brain caused by vascular obstruction, impaired vasodilation or increased blood viscosity leading to neuronal injury and parenchymal necrosis (Garosi et al., 2006; Higgins et al., 2006; Hillock et al., 2006; Wessmann et al., 2009; Giannuzzi et al., 2014). Depending on the size of the involved vessel, cerebral infarcts are distinct in territorial infarcts, associated with disease of superficial, large diameter blood vessels and lacunar infarcts, deriving from disease of small, intraparenchymal, penetrating arteries (Garosi et al., 2006). Clinical signs of focal ischemic encephalopathy are variable and ultimately related to the involved brain area (telencephalon; thalamus or midbrain; cerebellum; brainstem) (Hillock et al., 2006). Although a large percentage, more or less $40 \%$, of ischemic strokes have an unknown etiology, several underlying causes have been recognized in dogs and cats including, hypertension, endocrine, kidney, heart, metastatic diseases, parasitic thromboembolism (Garosi, 2010) and Evans' syndrome (Giannuzzi et al., 2014).

Focal ischemic encephalopathy is frequently diagnosed in companion animals and, in the last decade, it has been more commonly recognized likely because of both increased awareness of it as a potential neurologic disorder and increased availability of magnetic resonance imaging (MRI) and computed tomography (Dewey, 2003; Hillock et al., 2006). However, despite the large amount of medical data regarding ischemic stroke in dogs, description of such condition in wild canids is lacking in veterinary literature.

In this paper, the authors describe the clinical signs, MRI findings and follow up of a presumptive focal ischemic encephalopathy in a wild red fox (Vulpes vulpes) in Southern Italy.

\section{Case details}

A wild young male red fox (Vulpes vulpes) weighting $10 \mathrm{~kg}$ was found in the mountainous hinterland of Rome (Italy) with a heavily depressed mental status and unresponsive to the surrounding environment. The animal was able to stand, with pronounced right head tilt, showing no aggressiveness nor fear towards humans. The fox received a single dose of dexamethasone and amoxicillin-clavulanic acid by the first examiner veterinarian and three days later the animal was referred to the Pingry Veterinary Hospital of Bari with an improved reactivity towards the surrounding environment. On physical examination, no abnormalities were observed. Neurological examination revealed depression, circling to the left, right sided head tilt and decreased postural reactions on the left side. Ventromedial positional strabismus was the most reliable abnormality detectable on cranial nerves examination (Fig. 1). The menace response was questionable on both eyes. Neurological abnormalities suggested a multifocal encephalic neuroanatomic localization with right forebrain and central vestibular system involvement. Because of the lack of a reliable clinical history, creating an appropriate differential diagnosis list was not possible. 


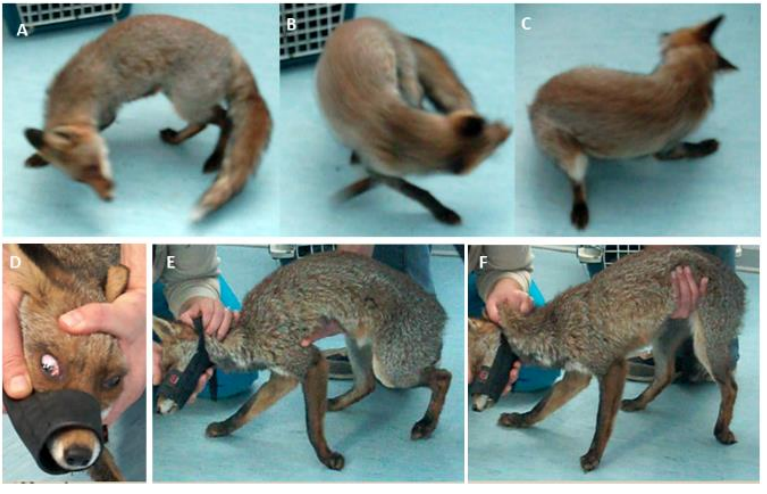

Fig. 1. Neurological examination. (A,B,C): Major neurological examination included depression, left circling with right head tilt. (D): Ventromedial positional strabismus on the right eye. $(\mathbf{E}, \mathbf{F})$ : decreased postural reactions on the left side. Neurological abnormalities suggested a multifocal encephalic neuroanatomic localization with right forebrain and central vestibular system involvement.

Brain MRI was performed using a 0.25 Tesla permanent magnet (ESAOTE VET-MR GRANDE, Esaote, Genoa, Italy) with the fox under general anesthesia. MRI sequences used included a Fast SE T2-W acquired in sagittal and transverse plane, a fluid attenuated inversion recovery (FLAIR) image, and a SE T1-W acquired in transverse plane before and after intravenous administration of paramagnetic contrast medium (Magnegita, gadopentetate dimeglumine $500 \mathrm{mmol} / \mathrm{mL}$, insight agents; $0.15 \mathrm{mmol} / \mathrm{kg} \mathrm{BW}$ ).

T2W and FLAIR images showed a sharply hyperintense, well demarcated lesion at the ventromedial surface of the right temporal lobe with focal involvement of the ventrolateral portion of mesencephalic tegmentum and adjacent caudo-ventrolateral portion of the right thalamus (Figs. 2, 3).

In the temporal lobe signal changes involved both gray and white matter with major involvement of cerebral cortex of the parahippocampal gyrus and ventral portion of right hippocampus. The lesion appeared isointense on T1-W images with mild and irregular enhancement after contrast medium administration (Fig. 2). No mass effect was evident. The distribution of the lesion matched the territory of the right caudal cerebral artery and its paramedian branches with possible involvement of caudal perforating arteries arising from basilar bifurcation. These findings were primarily suggestive of vascular ischemic lesion while inflammatory conditions were considered less likely. Protein levels ( $14 \mathrm{mg} / \mathrm{dl}$; reference interval: $<30 \mathrm{mg} / \mathrm{dl}$ ) and cell count ( 3 cell $/ \mu 1$; reference interval: $0-3$ cell $/ \mu 1$ ) of a CSF sample collected from the cerebellomedullary cistern were apparently normal. Fecal flotation test was positive for ascarids eggs and two consecutive Baermann tests were negative for strongyles and Crenosoma vulpis larvae.

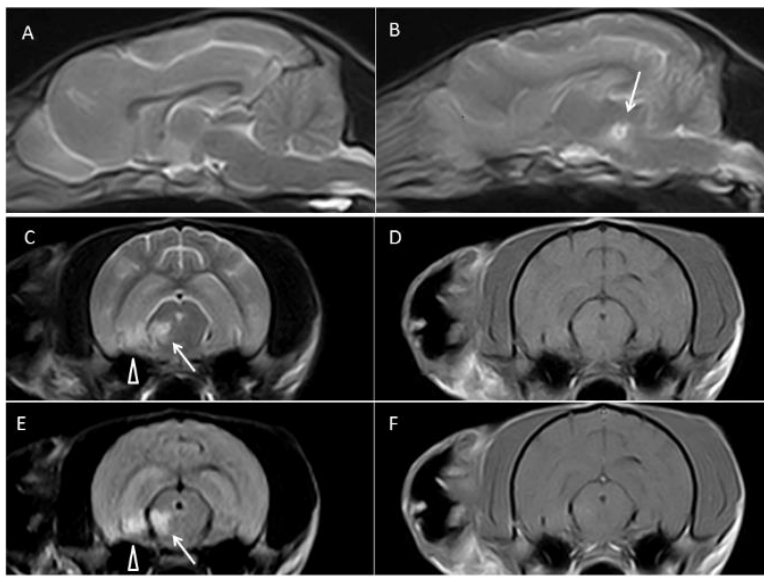

Fig. 2. First MRI of the brain. (A): midsagittal and (B): right parasagittal T2-weighted MRI images. (C): Transverse T2weighted, (D): T1-weighted, (E): FLAIR and (F): contrastenhanced T1-weighted MRI images obtained at the level of the rostral midbrain. T2W ad FLAIR images show a sharply hyperintense well demarcated lesion affecting the ventrolateral portion of mesencephalic tegmentum (arrows) and adjacent caudo-ventro-lateral portion of the right thalamus (not shown). The lesion extends to the medial surface of the right temporal lobe with involvement of cerebral cortex of the parahippocampal gyrus and ventral portion of right hippocampus (arrowheads).The lesion appears isointense on T1-W images with faint and irregular enhancement after contrast medium administration. No mass effect is evident.

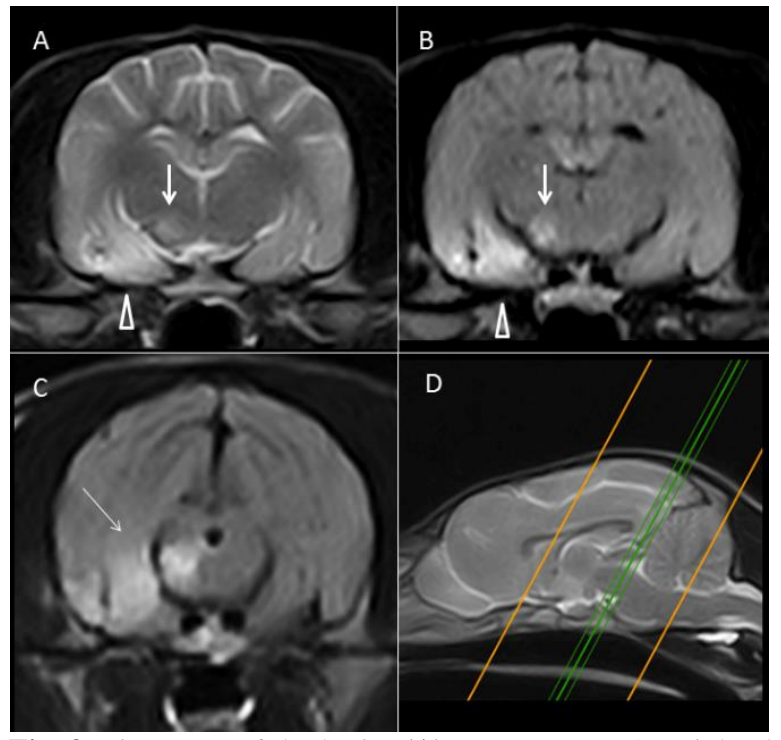

Fig. 3. First MRI of the brain. (A): Transverse T2-weighted and (B): FLAIR MRI images obtained at the level of the caudal thalamus. The mesencephalic lesion extended cranially with focal involvement of the caudo-ventro-lateral portion of the right thalamus (arrows). In the temporal lobe signal changes involved both gray and white matter of the parahippocampal gyrus. (C): Oblique-transverse FLAIR image at level of cross reference in image (D), showing in detail the mesencephalic and telencephalic lesion extension (thin arrow). 
Table 1. Hemato-biochemical findings.

\begin{tabular}{|c|c|c|c|c|}
\hline Analyte & Value & $\begin{array}{c}\text { Reference intervals for } \\
\text { juvenile Urocyon Littoralis }(\S)\end{array}$ & $\begin{array}{l}\text { Mean values for } \\
\text { Vulpes velox (\$D) }\end{array}$ & $\begin{array}{l}\text { Reference intervals } \\
\text { for adult dogs }(*)\end{array}$ \\
\hline $\operatorname{RBC}\left(\mathrm{x} 10^{6} / \mu \mathrm{l}\right)$ & 14.94 & $5.9-8.4$ & $9.46 \pm 0.92$ & $5.7-8.56$ \\
\hline $\mathrm{Hb}(\mathrm{g} / \mathrm{dl})$ & 17.6 & $11.4-16.2$ & $17.95 \pm 1.84$ & $14.1-21.2$ \\
\hline $\mathrm{HCT}(\%)$ & 61.9 & $37.4-54.2$ & $51.15 \pm 4.84$ & $39.0-59.2$ \\
\hline $\operatorname{MCV}(\mathrm{fl})$ & 41.4 & $57-71$ & $53.65 \pm 2.33$ & $63.1-72.6$ \\
\hline $\mathrm{MCH}(\mathrm{pg})$ & 11.8 & $17.6-20.7$ & $18.57 \pm 0.61$ & $21.8-25.4$ \\
\hline $\mathrm{MCHC}(\mathrm{g} / \mathrm{dl})$ & 28.4 & $27.7-34.5$ & $34.47 \pm 1.19$ & $33.3-36.8$ \\
\hline NRBC (/100 WBC) & 0 & $0-6$ & n.a. & $0-0$ \\
\hline $\mathrm{WBC}\left(\mathrm{x} 10^{3} / \mu \mathrm{l}\right)$ & 5.92 & $6.7-15.7$ & $5.05 \pm 1.354$ & $5.45-12.98$ \\
\hline Absolute segmented neutrophils $(/ \mu \mathrm{l})$ & 2424 & $3567-10836$ & n.a. & $3555-9314$ \\
\hline Absolute band neutrophils $(/ \mu \mathrm{l})$ & 0 & $0-110$ & n.a. & $0-286$ \\
\hline Absolute lymphocytes $(/ \mu \mathrm{l})$ & 1858 & $666-5217$ & n.a. & $1169-3810$ \\
\hline Absolute monocytes $(/ \mu \mathrm{l})$ & 550 & $66-1026$ & n.a. & $186-798$ \\
\hline Absolute eosinophils $(/ \mu \mathrm{l})$ & 1047 & $87-2882$ & n.a. & $104-1164$ \\
\hline Absolute basophils $(/ \mu \mathrm{l})$ & 41 & $0-429$ & n.a. & $0-106$ \\
\hline $\operatorname{PLT}\left(\times 10^{3} / \mu \mathrm{l}\right)$ & 418 & - & n.a. & $176-479$ \\
\hline $\operatorname{ALP}(\mathrm{U} / \mathrm{l})$ & 81 & $13-184$ & $53.43 \pm 76.25$ & $24-40$ \\
\hline $\operatorname{ALT}(\mathrm{U} / \mathrm{I})$ & 304 & $45-305$ & n.a. & $30-60$ \\
\hline $\operatorname{AST}(\mathrm{U} / \mathrm{l})$ & 52 & $27-154$ & n.a. & $19-29$ \\
\hline CK (U/l) & 142 & $94-3045$ & n.a. & $49-126$ \\
\hline $\mathrm{TP}(\mathrm{g} / \mathrm{dl})$ & 6.5 & $4.9-7.6$ & $6.28 \pm 0.63$ & $5.9-71$ \\
\hline Albumin (g/dl) & 2.6 & $2.6-3.8$ & $2.83 \pm 0.26$ & $3.0-3.4$ \\
\hline Globulin (g/dl) & 3.9 & $2.2-4.4$ & $3.45 \pm 0.68$ & $2.6-3,4$ \\
\hline Total bilirubin (mg/dl) & 0.2 & $0.0-0.2$ & $0.17 \pm 0.16$ & $0.13-0.2$ \\
\hline BUN (mg/dl) & 35 & $10-36$ & $23.35 \pm 6.77$ & $21-48$ \\
\hline Creatinine (mg/dl) & 0.82 & $0.4-1.0$ & $0.79 \pm 0.63$ & $0.76-1.09$ \\
\hline Cholesterol (mg/dl) & 200 & $107-197$ & n.a. & $175-248$ \\
\hline Glucose (mg/dl) & 120 & $91-199$ & $100.87 \pm 48.61$ & $100-109$ \\
\hline Calcium (mg/dl) & 10.2 & $8.0-10.3$ & $11.57 \pm 1.78$ & $9.5-10.7$ \\
\hline Phosphorus (mg/dl) & 6.2 & $3.3-8.8$ & $5.69 \pm 1.26$ & $2.1-3.8$ \\
\hline Bicarbonate (mEq/l) & 22.9 & $10-21$ & n.a. & $17.4-24.2$ \\
\hline Chloride (mEq/l) & 113 & $105-118$ & n.a. & $110-115$ \\
\hline Potassium (mEq/l) & 5.0 & $3.7-5.5$ & $5.0 \pm 0.66$ & $4.2-4.5$ \\
\hline Sodium $(\mathrm{mEq} / \mathrm{l})$ & 147 & $141-154$ & $148.78 \pm 4.64$ & $145-148$ \\
\hline
\end{tabular}

(WBC): white blood cell; (RBC): red blood cell; (Hb): hemoglobin; (HCT): hematocrit; (MCV): mean corpuscular volume; $(\mathrm{MCH})$ : mean corpuscular hemoglobin; (MCHC): mean corpuscular hemoglobin concentration; (NRBC): nucleated red blood cell; (PLT): platelets; (ALP): alkaline phosphatase; (ALT): alanine transaminase; (AST): aspartate transaminase; (CK): creatine kinase; (TP): total protein; (BUN): blood urea nitrogen; (n.a.): not available.

(§) Biochemical and hematologic reference intervals for the island fox (Urocyon littoralis) (Inoue et al., 2012).

(II) Mean values for haematology and serum biochemistry from a population of swift foxes (Vulpes velox) (Mainka, 1988).

(*) Reference values provided by a certified veterinary laboratory (San Marco Veterinary Laboratory, Padova, Italy)

Complete blood (cell) count (CBC), and biochemical profile were also carried out. Hemato-biochemical analysis apparently revealed erythrocytosis, microcytosis, hypochromic red blood cells and mild neutropenia (Table 1 - hemato-biochemical results were compared with the reference values of the island fox and dogs, and with mean values available for Vulpes velox).

Within 30 days of hospitalization the fox showed progressive clinical improvement without medical therapy. A second neurological examination revealed normalization of vestibular signs and left side postural reactions with residual mild left circling.
A MRI of the brain was repeated using the same sequence protocol. The second imaging examination showed significant reduction in size of the primary lesions and fluid replacement of T2 and FLAIR hyperintense mesencephalic areas previously detected (Fig. 4).

Based on MRI pattern of distribution and evolution of the lesions, spontaneous improvement of neurological signs and CSF analysis, a presumptive diagnosis of ischemic infarct in the territory of right caudal cerebral artery and its collateral branches was made. Involvement of right caudal perforating arteries arising from basilar bifurcation was also considered. 


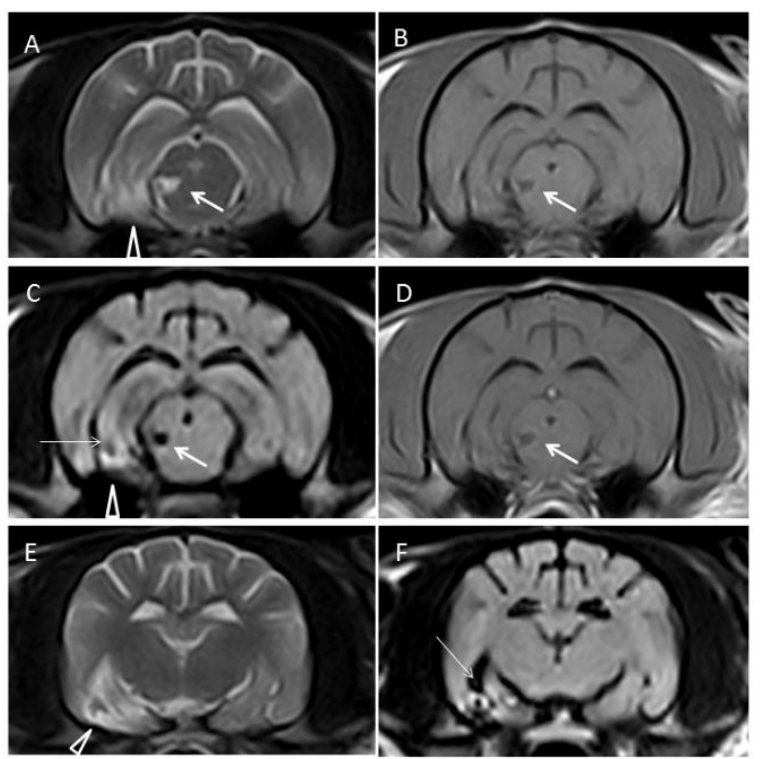

Fig. 4. Repeat MRI of the brain acquired 30 days after the first examination. (A): Transverse T2-weighted, (B): T1weighted, (C): FLAIR and (D): contrast-enhanced T1weighted MRI images obtained at the level of the rostral midbrain. There is significant reduction in size of the primary lesions and fluid replacement of T2 and FLAIR hyperintense mesencephalic areas previously detected (arrows). (E): Transverse T2-weighted and (F): FLAIR MRI images obtained at the level of the caudal thalamus showing normal parenchymal signal intensity of the thalamic area previously involved by the lesion. Slight residual hyperintensity is observed at the level of parahippocampal gyrus (arrowheads) with fluid signal at the level of the ventral portion of right hippocampus (thin arrows).

\section{Discussion}

Canidae is one of the most studied mammalian groups. From comparative studies on their neuroanatomy it has been clarified that the external cerebrum morphology of the modern Canidae is extremely uniform and, except for small differences in the shape and size of frontal gyri (sigmoid and proreal gyrus), characterized by lack of important differences between the genera (Radinsky, 1969, 1973, 1978; Lyras and Van Der Geer, 2003). Such uniformity can also be observed from the comparison of cross-sectional anatomy of the red fox and dog brain as revealed by previous MRI studies in both species (Kassab and Bahgat, 2007; Leigh et al., 2008).

As reported in dogs (Garosi et al., 2006), the imaging findings (shape and distribution of the lesions, absence of mass effect, signal intensity and evolution of the lesions) in the fox herein examined were suggestive of ischemic infarct in the territory of the right caudal cerebral artery (CCA).

In dogs, the CCAs arise from the caudal communicant arteries of the cerebral arterial circle (circle of Willis) and run caudo-laterally supplying blood to the caudal and medial surface of each telencephalic hemisphere.
Along their course the CCAs give rise to small branches to the ventro-medial portion of temporal lobes, to the caudo-lateral part of the thalamus and to the lateral mesencephalon (perforating arteries) (Barone, 2003; Garosi et al., 2006; Hillock et al., 2006).

The same territorial distribution of the CCAs have been proven in detailed anatomical studies on the vascular brain anatomy in the red fox and pampas fox (Pseudalopex gumnocercus).

In particular, specific collateral CCA branches to the piriform lobe, parahippocampal gyrus, thalamus and mesencephalon have been described in such species (Depedrini and Campos, 2003, 2007; Ozudogru et al., 2012)

The distribution of the lesions observed on MRI in the ventrolateral mesencephalon, ventrolateral caudal thalamus, piriform lobe and para-hippocampal gyrus confirmed the course of the collateral CCA branches as also described in foxes (Depedrini and Campos, 2003, 2007; Ozudogru et al., 2012) and matched the most commonly affected regions reported in dogs with CCA infarction (Garosi et al., 2006).

However, for thalamic and midbrain lesions, concomitant involvement of caudal perforating arteries arising from basilar bifurcation could not be ruled out (Garosi et al., 2006).

In this fox, the presence of vestibular signs resulted apparently unsolved and speculative.

In cats, unilateral experimentally-induced mesencephalic lesions, resulted in lateral tilt of the head toward the opposite side, while bilateral lesions induced dorsiflexion of the head (Fukushima et al., 1987). Anatomical basis associated with these abnormal head posture involve dysfunction of the interstitial nucleus of Cajal (INC) (in the rostral midbrain adjacent to the periaqueductal gray matter), interstitiospinal fibres (that run in the medial longitudinal fasciculus) (Nyberg-Hansen, 1966) and their control on the rostral cervical muscles (Fukushima et al., 1987).

In particular, unilateral induced lesion in INC or in its descending fibres up to the caudal region of mesencephalon, cause an increase in activity of the major ipsilateral dorsal neck muscles and in the contralateral obliquus capitis caudalis resulting in controlateral head tilt (Fukushima et al., 1985, 1987; Kavaklis et al., 1992).

Bilateral lesions induce activation of dorsal neck muscles producing dorsiflexion of the head (Fukushima et al., 1987; Fukushima-Kudo et al., 1987).

In dogs, mesencephalic dysfunction has been related with different abnormal neck and head posture (Garosi et al., 2006; Goncalves et al., 2011, Canal et al., 2015). In dogs with ventrolateral thalamic infractions, the lesions were associated with mesencephalic involvement resulting in controlateral or ipsilateral 
head tilt as prevailing vestibular sing (Goncalves et al., 2011).

Moreover, in two dogs with intracranial expansive lesions exerting bilateral compression of dorsal mesencephalon, permanent neck extension (retrocollis) has been observed and attributed to bilateral INC dysfunction (Canal et al., 2015). Interestingly, neurological signs detected in this fox perfectly reflected those reported for ventrolateral thalamic infractions with mesencephalic involvement in dogs (Garosi et al., 2006; Goncalves et al., 2011).

In this fox, as well as in previously reported canine cases, while compulsive circling and controlateral proprioceptive deficit were well explained by prosencephalic lesions, the neuroanatomic explanation of the concomitant ipsilateral vestibular signs is consistent with unilateral involvement of nucleus of Cajal in the rostral mesencephalon.

Thalamic dysfunction is also suspected to cause vestibular signs in dogs, especially after acute lesions (de Lahunta and Glass, 2009). The pathways for conscious balance perception involving a relay from a thalamic nucleus seems implicated in the vestibular thalamic syndrome (Brandt and Dieterich, 1999).

In attempt to identify possible underling risk factors for brain infarction, hemato-biochemical analysis were performed. Unfortunately, normal reference values for red foxes (Vulpes vulpes) are not available in veterinary medical literature.

To the author's knowledge hemato-biochemical reference intervals for wild foxes are available only for the island fox (urocyon littoralis) (Inoue et al., 2012). In addition hematologic and serum chemistry mean values are available for the species Vulpes velox (Mainka, 1988). From the analysis of hematobiochemical abnormalities detected in our fox using the reference values of the island fox and dogs, and by the comparison with mean values available for Vulpes velox species, erythrocytosis associated with microcytosis and hypochromic red blood cells and mild neutropenia were apparently detectable (Table 1). However, taking into account the possible variability existing among these different species, such abnormal findings were considered of doubtful interpretation.

Thus, in this fox the possible cause of brain infarction remains unclear due to the lack of further diagnostic evaluations (blood pressure measurement, thoracic and abdominal imaging evaluation, urinalysis, endocrine tests) and the equivocal relevance of hematobiochemical abnormalities.

Finally, this case is the first report of a presumptive thalamic and midbrain infarction in a fox showing multifocal encephalic syndrome with predominant vestibular dysfunction. This case suggests not only an anatomic but also a neurophysiologic analogy between dogs, cats and foxes.

\section{Acknowledgments}

The authors wish to thank all the staff of the Pingry Veterinary Hospital of Bari, Italy for their assistance with data collection.

\section{Conflict of interest}

The authors declare that there is no conflict of interests.

\section{References}

Barone, R. 2003. Anatomia comparata dei mammiferi domestici: Neurologia, Vol. 6, $3^{\text {rd }}$ ed. Edagricole, Bologna.

Brandt, T. and Dieterich, M. 1999. The vestibular cortex: its locations, functions, and disorders. Ann. N. Y. Acad. Sci. 871, 293-312.

Canal, S., Baroni, M., Falzone, C., De Benedictis, G.M. and Bernardini, M. 2015. Dorsal midbrain syndrome associated with persistent neck extension: clinical and diagnostic imaging findings in two dogs. Can. Vet. J. 56, 1261-1265.

de Lahunta, A. and Glass, E. 2009. Veterinary Neuroanatomy and Clinical Neurology. $3^{\text {rd }}$ ed. Elsevier, St. Louis, Miss, USA, pp: 324.

Depedrini, J.S. and Campos, R. 2003. A systematic study of the brain base arteries in the pampas fox (Dusicyon gymnocercus). Braz. J. Morphol. Sci. 20, 181-188.

Depedrini, J.S. and Campos, R. 2007. Systematization, distribution and territory of the caudal cerebral artery on the surface of the brain in pampas foxes (Pseudalopex gymnocercus). Braz. J. Morphol. Sci. 24, 126-136.

Dewey, C.W. 2003. Vascular encephalopathies in the dog and cat. Proc. $21^{\text {st }}$ ACVIM Forum, pp: 398-400.

Fukushima, K., Fukushima, J. and Terashima, T. 1987. The pathways responsible for the characteristic head posture produced by lesions of the interstitial nucleus of Cajal in the cat. Exp. Brain Res. 68, 88102.

Fukushima, K., Takahashi, K., Kudo, J. and Kato, M. 1985. Interstitialvestibular interaction in the control of head posture. Exp. Brain Res. 57, 264-270.

Fukushima-Kudo, J., Fukushima, K. and Tashiro, K. 1987. Rigidity and dorsiflexion of the neck in progressive supranuclear palsy and the interstitial nucleus of Cajal. J. Neurol. Neurosurg. Psych. 50, 1197-1203.

Garosi, L., McConnell, J.F., Platt, S.R., Barone, G., Baron, J.C., de Lahunta, A. and Schatzberg, S.J. 2006. Clinical and topographic magnetic resonance characteristics of suspected brain infarction in 40 dogs. J. Vet. Intern. Med. 20, 311-321.

Garosi, L.S. 2010. Cerebrovascular disease in dogs and cats. Vet. Clin. North Am. Small Anim. Pract. 40, 65-79.

Giannuzzi, A.P., DeSimone, A., Ricciardi, M. and Gernone, F. 2014. Presumptive Ischemic Brain 
Infarction in a Dog with Evans' Syndrome. Case Reports in Veterinary Medicine. Volume 2014, Article ID 456524.

Goncalves, R., Carrera, I., Garosi, L., Smith, P.M., Fraser McConnell, J. and Penderis, J. 2011. Clinical and topographic magnetic resonance imaging characteristics of suspected thalamic infarcts in 16 dogs. Vet. J. 188, 39-43.

Higgins, M.A., Rossmeisl, J.H. Jr. and Panciera, D.L. 2006. Hypothyroid-associated central vestibular disease in 10 dogs: 1999-2005. J. Vet. Intern. Med. 20, 1363-1369.

Hillock, S.M., Dewey, C.W., Stefanacci, J.D. and Fondacaro, J.V. 2006. Vascular encephalopathies in dogs: incidence, risk factors, pathophysiology, and clinical signs. Compend. Contin. Educ. Pract. Vet. 28, 196-207.

Inoue, H., Clifford, D.L., Vickers, T.W., Coonan, T.J., Garcelon, D.K. and Borjesson, D.L. 2012. Biochemical and hematologic reference intervals for the endangered island fox (Urocyon littoralis). J. Wildl. Dis. 48, 583-592.

Kassab, A. and Bahgat, H. 2007. Magnetic Resonance Imaging and cross-sectional anatomy of the brain of the red fox (Vulpes vulpes).Vet. Med. J. Giza. 55, 779-786.

Kavaklis, O., Shima, F., Kato, M. and Fukui, M. 1992. Ipsilateral pallidal control of the sternocleidomastoid muscle of cats: Relationship to the side of thalamotomy for torticollis.
Neurosurgery 30, 724-730.

Leigh, E.J., Mackillop, E., Robertson, I.D. and Hudson, L.C. 2008. Clinical anatomy of the canine brain using magnetic resonance imaging. Vet. Radiol. Ultrasound. 49, 113-121.

Lyras, G.A. and Van Der Geer, A.A.E. 2003. External brain anatomy in relation to phylogeny of Caninae (Carnivora: Canidae). Zool. J. Linn. Soc. 138, 505522.

Mainka, S.A. 1988. Hematology and serum biochemistry of captive swift foxes (Vulpes velox). J. Wildl. Dis. 24, 71-74.

Nyberg-Hansen, R. 1966. Sites of termination of interstitiospinal fibers in the cat. An experimental study with silver impregnation methods. Arch. Ital. Biol. 104, 98-111.

Ozudogru, Z., Can, M. and Balkaya, H. 2012. MacroAnatomical Investigation of the Cerebral Arterial Circle (Circle of Willis) in Red Fox (Vulpes vulpes Leunnoleus, 1758). J. Anim. Vet. Adv. 11, 28612864.

Radinsky, L.B. 1969. Outlines of canid and felid brain evolution. Ann. N. Y. Acad. Sci. 167, 277-288.

Radinsky, L.B. 1973. Evolution of the canid brain. Brain Behav. Evol. 7, 169-202.

Radinsky, L.B. 1978. The evolutionary history of dog brains. Museologia 10, 25-29.

Wessmann, A., Chandler, K. and Garosi, L. 2009. Ischaemic and haemorrhagic stroke in the dog. Vet. J. 180, 290-303. 